Article

\title{
Conjugate Addition of Nucleophiles to the Vinyl Function of 2-Chloro-4-vinylpyrimidine Derivatives
}

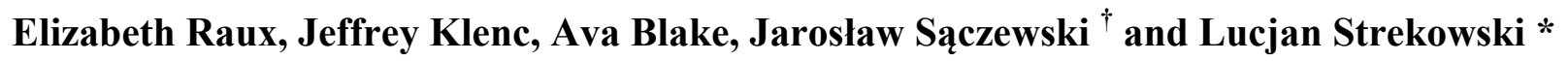 \\ Department of Chemistry, Georgia State University, Atlanta, Georgia 30302-4098, USA \\ $\dagger$ On leave of absence from Department of Chemical Technology of Drugs, Medical University of \\ Gdańsk, Al. Gen. Hallera 107, 80-416 Gdańsk, Poland. \\ * Author to whom correspondence should be addressed; E-Mail: lucjan@gsu.edu.
}

Received: 2 March 2010; in revised form: 9 March 2010 / Accepted: 18 March 2010 /

Published: 19 March 2010

\begin{abstract}
Conjugate addition reaction of various nucleophiles across the vinyl group of 2-chloro-4-vinylpyrimidine, 2-chloro-4-(1-phenylvinyl)pyrimidine and 2-chloro-4-vinylquinazoline provides the corresponding 2-chloro-4-(2-substituted ethyl)pyrimidines and 2-chloro-4-(2-substituted ethyl)quinazolines. Treatment of these products, without isolation, with $\mathrm{N}$-methylpiperazine results in nucleophilic displacement of chloride and yields the corresponding 2,4-disubstituted pyrimidines and quinazolines.
\end{abstract}

Keywords: conjugate addition; vinylpyrimidine; vinylquinazoline

\section{Introduction}

Few addition reactions across the vinyl group of vinyl-substituted heterocyclic compounds are known. Photoirradiation of 2-vinylpyridine in acidic alcohol affords alkyl 2-(2-pyridyl)ethyl ether [1]. The treatment of 2-vinylpyridine or 4-vinylpyridine under acidic conditions with various amines yield the corresponding aminoethyl-substituted pyridines [2]. Ethyl methyl sulfones are produced upon treatment of vinyl-substituted pyridines, pyrimidines and other diazines with sodium methanesulfinate under acidic conditions [3]. The reaction of indole with the vinyl-substituted substrates yields 2-(indol3-yl)ethyl derivatives when conducted under acidic conditions [4]. On the other hand, 2-(indol-1yl)ethyl substituted heteroaromatic compounds are produced for the reactions conducted under basic conditions at elevated temperatures [4]. Finally, various alkylthiols and arylthiols undergo a facile 
addition reaction with 4-vinylquinazoline to give the corresponding 4-[2-(alkyl/arylthio)ethyl]quinazolines [5].

This report pertains to the conjugate addition reaction of N-, O-, S-, and C-centered nucleophiles across the vinyl function of 2-chloro-4-vinylpyrimidine (2), 2-chloro-4-(1-phenylvinyl)pyrimidine (3), and 2-chloro-4-vinylquinazoline (19). These vinyl-bearing substrates are readily available by the addition reaction of a vinyllithium reagent to the formal $\mathrm{C} 4=\mathrm{N} 3$ bond of 2-chloropyrimidine and 2chloroquinazoline, followed by aromatization of the resultant adduct [4-7]. Another approach to similar compounds involves dehydration of an alcohol to the vinyl group [7]. The study described in this paper provides a facile modification of the pyrimidine and quinazoline systems with diverse substituents. More specifically, the selective nucleophile addition reaction across the vinyl group can be followed by nucleophilic displacement reaction of the 2-chloride substituent in the intermediate product to give a disubstituted pyrimidine derivative. Similar compounds have been shown to interact selectively with various serotonin receptors of the central nervous system (CNS) and have shown promise for the development as practical CNS drugs [6,8-10].

\section{Results and Discussion}

Improved procedures for the preparation of 2-chloro-4-vinylpyrimidine (2, Scheme 1) [11] and 2chloro-4-vinylquinazoline (19, Scheme 4) [5] have been reported by us recently [6]. 2-Chloro-4-(1phenylvinyl)pyrimidine (3, Scheme 1$)$ is a new compound. For the synthesis of $\mathbf{3}, \alpha$-bromostyrene was allowed to react with $n$-butyllithium and this generated lithium reagent was treated with 2chloropyrimidine (1). The resultant adduct was then aromatized by the reaction with 2,3-dichloro-5,6dicyanoquinone (DDQ) to give the desired product 3.

Scheme 1. Synthesis of pyrimidines 5-12.

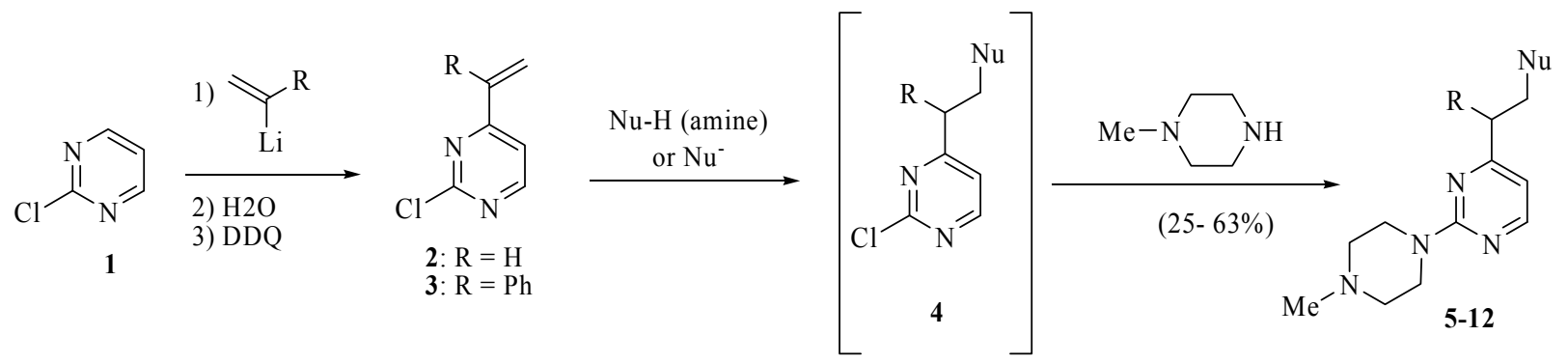

\begin{tabular}{cccccc}
\hline$\#$ & $\mathrm{R}$ & $\mathrm{Nu}$ & $\mathrm{R}$ & $\mathrm{Nu}$ \\
\hline $\mathbf{5}$ & $\mathrm{H}$ & $\mathrm{NMe}_{2}$ & $\mathbf{9}$ & $\mathrm{H}$ & $\mathrm{OMe}$ \\
$\mathbf{6}$ & $\mathrm{H}$ & $\mathrm{HN}^{-\mathrm{Bu}^{n}}$ & $\mathbf{1 0}$ & $\mathrm{H}$ & $\mathrm{SEt}$ \\
$\mathbf{7}$ & $\mathrm{H}$ & $\mathbf{1 1}$ & $\mathrm{Ph}$ & $\mathrm{HNCH}_{2} \mathrm{Ph}^{t}$ \\
$\mathbf{8}$ & $\mathrm{HN}-\mathrm{Bu}^{t}$ & $\mathbf{1 2}$ & $\mathrm{Ph}$ & $\mathrm{SEt}$ \\
\hline
\end{tabular}

As can be seen from Scheme 1, a variety of $N$-, $O$-, and $S$-centered nucleophiles undergo selective addition reactions across the vinyl function of $\mathbf{2}$ and $\mathbf{3}$. A subsequent treatment of the crude reaction mixture containing the corresponding adducts 4 with $N$-methylpiperazine furnished the corresponding 
products 5-12. The yields ranged from a low of $25 \%$ for the ethanethiol derivative $\mathbf{1 0}$ to a high of $63 \%$ for the benzylamino derivative 8. It should be noted that these yields are for the analytically pure products obtained in the indicated two-step transformations starting with 2-chloropyrimidine (1). In all cases, compounds 5-12 were the major products that were easily isolated by chromatography. The reaction also produced many minor products that could not be isolated for identification and a substantial amount of tar. The high selectivity of the first addition reaction across the vinyl function was consistent with a GC-MS analysis of all crude mixtures containing 4, which indicated the presence of the chlorine atom in the major product. In one case the intermediate product $4(\mathrm{R}=\mathrm{Ph}, \mathrm{Nu}=\mathrm{MeO})$ was isolated and characterized.

The chemistry of Scheme 1 was conducted using equimolar amounts of the vinylpyrimidine and nucleophile substrates. It was shown that the rate of the vinyl addition reaction is greater than that of the chloride displacement. Additional evidence for the selective nucleophile addition is presented in Scheme 2 for the treatment of 2 with 0.55 equiv. of ethylamine followed by standard treatment of the presumed intermediate product 13 with $N$-methylpiperazine. The final product 14 was the only major component of the crude mixture and was isolated in an analytically pure form in a $38 \%$ yield.

Scheme 2. Synthesis of pyrimidine 14.

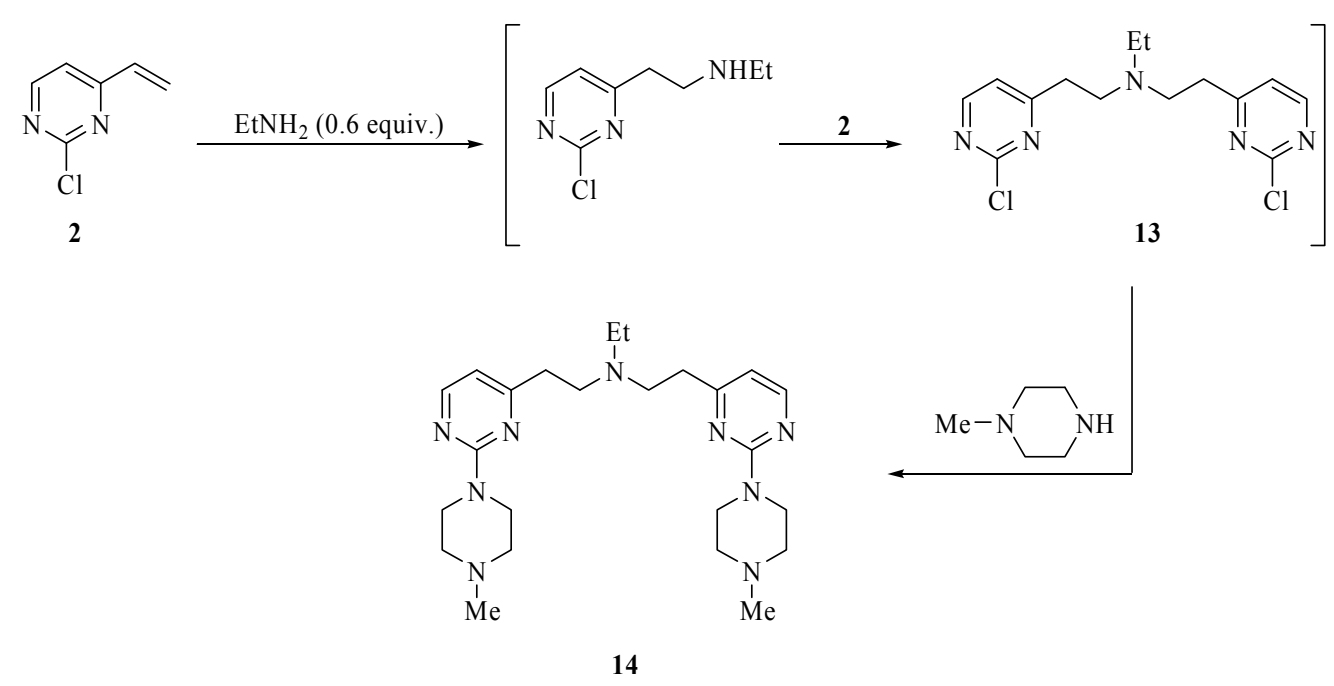

The treatment of $\mathbf{2}$ or $\mathbf{3}$ with lithium reagents such as methyllithium and phenyllithium produced a large number of products, none of them major. These reactions were not simplified when conducted in the presence of cuprous iodide. However, analogous reactions with the corresponding Grignard reagents gave less complicated mixtures, which upon treatment with $N$-methylpiperazine yielded the corresponding products 15-17 that were isolated and characterized. Interestingly, the low yields of 15-17 $(<10 \%)$ were increased to the range $40-77 \%$ when the initial addition reactions were conducted in the presence of cuprous iodide. Under the optimized conditions the ratio of Grignard reagent to $\mathrm{CuI}$ is $2: 1$. The observed effect of $\mathrm{CuI}$ is consistent with a mechanism the initial step of which involves single electron transfer (SET) from a cuprate intermediate product to the vinylpyrimidine substrate $[11,12]$. It is not certain, however, whether a SET process is operative in the addition reaction of heteroatom-centered nucleophiles. The structures of 15-17 are fully consistent with their high- 
resolution mass, ${ }^{1} \mathrm{H}-\mathrm{NMR}$, and ${ }^{13} \mathrm{C}-\mathrm{NMR}$ spectra. In particular, the absorption pattern for a propyl group, observed in the ${ }^{1} \mathrm{H}-\mathrm{NMR}$ spectra of $\mathbf{1 5}$ and $\mathbf{1 7}$, shows the selective addition of the methyl Grignard reagent to the vinyl function of $\mathbf{2}$ and $\mathbf{3}$, respectively.

Scheme 3. Synthesis of pyrimidines 15-17.

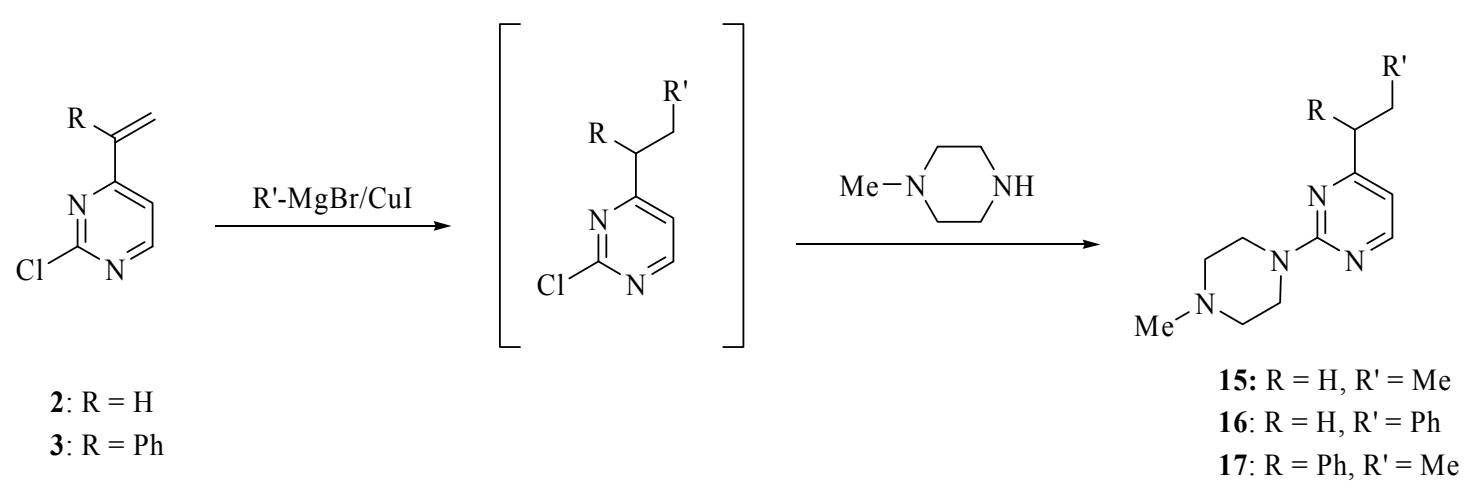

The synthesis of 2-chloro-4-vinylquinazoline (19) based on the addition reaction of vinyllithium with 2-chloroquinazoline (18, Scheme 4) has been reported by us recently [6]. We now report that, as with vinylpyrimidines, the addition reaction of nucleophiles with the vinyl function of 19 is highly selective. The presence of a chlorine atom in the intermediate adducts could be observed by mass spectrometry. As with the pyrimidines, treatment of the intermediate products with $N$-methylpiperazine furnishes the corresponding disubstituted quinazolines.

Scheme 4. Synthesis of quinazolines 20-26.

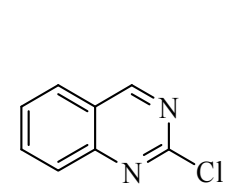

18
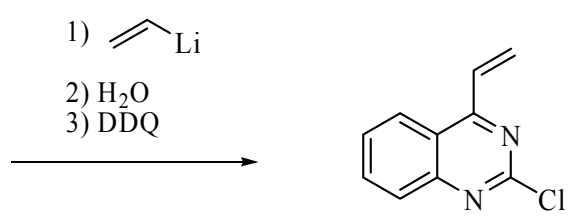

19

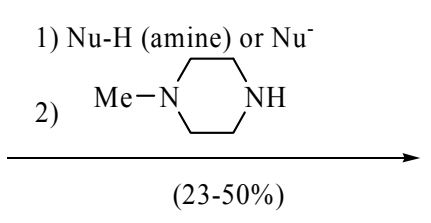

(23-50\%)<smiles>CN1CCN(c2nc(CC[AlH2])c3ccccc3n2)CC1</smiles>

\begin{tabular}{cccc}
\hline$\#$ & $\mathrm{Nu}$ & $\#$ & $\mathrm{Nu}$ \\
\hline $\mathbf{2 0}$ & $\mathrm{NMe}_{2}$ & $\mathbf{2 4}$ & $\mathrm{OMe}$ \\
$\mathbf{2 1}$ & $\mathrm{HN}-\mathrm{Bu}^{n}$ & $\mathbf{2 5}$ & $\mathrm{SEt}$ \\
$\mathbf{2 3}$ & $\mathrm{HNCH}{ }_{2} \mathrm{Ph}$ & $\mathbf{2 6}$ & $\mathrm{SPh}$ \\
\hline
\end{tabular}




\section{Experimental}

\subsection{General}

All organometallic reactions were conducted under a nitrogen atmosphere in tetrahydrofuran distilled from sodium benzophenone ketyl immediately before use. Final products were purified on a chromatotron using silica gel-coated rotors $(2.0 \mathrm{~mm})$. Hydrobromide and hydrochloride salts of the piperazine products were obtained by using a general procedure $[9,10]$ and the salts were crystallized from $95 \%$ ethanol. In several cases it was necessary to dilute the ethanolic solution with ether to induce crystallization. Melting points are not corrected. ${ }^{1} \mathrm{H}-\mathrm{NMR}$ and ${ }^{13} \mathrm{C}$-NMR spectra were recorded at $400 \mathrm{MHz}$ and $100 \mathrm{MHz}$, respectively, on a Bruker Avance insturment.

\subsection{Organometallic reagents}

n-Butyllithium (2.5 $\mathrm{M}$ in hexanes), and tert-butyllitium (1.7 $\mathrm{M}$ in pentane) were commercial reagents. Vinyllithium was generated by the reaction of tetravinyltin with tert-butyllithium as previously described $[6,13]$. Briefly, a solution of tetravinyltin $(0.7 \mathrm{~mL}, 3.2 \mathrm{mmol})$ in tetrahydrofuran $(10 \mathrm{~mL})$ was treated dropwise at $-70{ }^{\circ} \mathrm{C}$ for $5 \mathrm{~min}$ with tert-butyllithium $(7.4 \mathrm{~mL}, 12.6 \mathrm{mmol})$, and the mixture was stirred for an additional $10 \mathrm{~min}$ at $-70{ }^{\circ} \mathrm{C}$ before use. $\alpha$-Lithiostyrene was generated by dropwise addition of $n$-butyllithium $(1.2 \mathrm{~mL}, 3.0 \mathrm{mmol})$ to a stirred solution of $\alpha$-bromostyrene $(0.43 \mathrm{~mL}, 3.0 \mathrm{mmol})$ in tetrahydrofuran $(3.0 \mathrm{~mL})$ at $-70{ }^{\circ} \mathrm{C}$ and the mixture was kept at $-70{ }^{\circ} \mathrm{C}$ for $5 \mathrm{~min}$ before use. Methylmagnesium bromide (3.0 $\mathrm{M}$ in diethyl ether), and phenylmagnesium bromide (1.0 $\mathrm{M}$ in tetrahydrofuran) were commercial reagents.

2-Chloro-4-vinylpyrimidine (2). This compound was synthesized as reported by us previously [7] and characterized as follows (this characterization has not been published in detail previously): an oil, yield 50\%; ${ }^{1} \mathrm{H}-\mathrm{NMR}\left(\mathrm{CDCl}_{3}\right): \delta 8.57(\mathrm{~d}, J=5.0 \mathrm{~Hz}, 1 \mathrm{H}), 7.22(\mathrm{~d}, J=5.0 \mathrm{~Hz}, 1 \mathrm{H}), 6.70(\mathrm{~m}, 1 \mathrm{H}), 6.52(\mathrm{~m}$, $1 \mathrm{H}), 5.80(\mathrm{~m}, 1 \mathrm{H}) ;{ }^{13} \mathrm{C}-\mathrm{NMR}\left(\mathrm{CDCl}_{3}\right): \delta 165.5,161.6,159.9,133.9,125.3,116.5$. High-resolution MS (ESI, positive ion mode): calcd. for $\mathrm{C}_{6} \mathrm{H}_{5}{ }^{35} \mathrm{ClN}_{2}\left(\mathrm{M}^{+}+1\right), \mathrm{m} / \mathrm{z} 141.0218$; found $\mathrm{m} / \mathrm{z} 141.0220$.

2-Chloro-4-(1-phenylvinyl)pyrimidine (3). A solution of $\alpha$-lithiostyrene $(3.0 \mathrm{mmol})$ in tetrahydrofuran $(3 \mathrm{~mL})$ was generated as described above. This solution was treated at once with one portion of a solution of 2-chloropyrimidine $(\mathbf{1}, 0.21 \mathrm{~g}, 1.8 \mathrm{mmol})$ in tetrahydrofuran $(2 \mathrm{~mL})$. After stirring for an additional $5 \mathrm{~min}$ at $-70^{\circ} \mathrm{C}$ the mixture was quenched with water $(0.1 \mathrm{~mL})$ in tetrahydrofuran $(1 \mathrm{~mL})$, and the stirring was continued while the temperature was allowed to rise to $0{ }^{\circ} \mathrm{C}$. After treatment with a solution of 2,3-dichloro-5,6-dicyanoquinone (DDQ, $0.7 \mathrm{~g}, 3 \mathrm{mmol}$ ) in tetrahydrofuran $(4 \mathrm{~mL})$ the mixture was stirred at $23{ }^{\circ} \mathrm{C}$ for $5 \mathrm{~min}$ and then cooled to $0{ }^{\circ} \mathrm{C}$, treated with a cold solution of sodium hydroxide ( $4 \mathrm{M}, 2 \mathrm{~mL}, 8 \mathrm{mmol})$ and extracted at $0{ }^{\circ} \mathrm{C}$ with hexanes/tetrahydrofuran $(1: 1,20 \mathrm{~mL})$. The extract was dried over magnesium sulfate, filtered and concentrated under reduced pressure. The residue was purified by chromatography eluting with hexanes/dichloromethane (1:1) to provide 3 as colorless oil in 58\% yield $(0.23 \mathrm{~g}) ;{ }^{1} \mathrm{H}-\mathrm{NMR}\left(\mathrm{CDCl}_{3}\right): \delta 8.53(\mathrm{~d}, J=5.2 \mathrm{~Hz}, 1 \mathrm{H}), 7.40(\mathrm{~m}, 3 \mathrm{H}), 7.31$ $(\mathrm{m}, 2 \mathrm{H}), 7.09(\mathrm{~d}, J=5.2 \mathrm{~Hz}, 1 \mathrm{H}), 6.48(\mathrm{~m}, 1 \mathrm{H}), 5.77(\mathrm{~m}, 1 \mathrm{H}) ;{ }^{13} \mathrm{C}-\mathrm{NMR}\left(\mathrm{CDCl}_{3}\right): \delta 167.7,161.5$, 
159.8, 145.9, 138.1, 128.6, 128.5, 128.4, 123.1, 117.5. High-resolution MS (ESI, positive ion mode): calcd. for $\mathrm{C}_{12} \mathrm{H}_{10}{ }^{35} \mathrm{ClN}_{2}\left(\mathrm{M}^{+}+1\right), \mathrm{m} / \mathrm{z} 217.0533$; found $\mathrm{m} / \mathrm{z} 217.0526$.

3.3. General procedure for the syntheses of $\mathbf{4}(R=P h, N u=O M e$, Scheme 1$)$ and $\mathbf{5}-\mathbf{1 2}$

A solution of 2 or $3(0.4 \mathrm{mmol})$ in toluene $(3.0 \mathrm{~mL})$ was treated with nucleophile (amine, MeONa, EtSNa, $\mathrm{PhSNa}, 0.4 \mathrm{mmol}$ ). The mixture was stirred at room temperature overnight or at $90{ }^{\circ} \mathrm{C}$ for $2 \mathrm{~h}$, then cooled to room temperature, basified with sodium carbonate and extracted with ethyl ether $(3 \times 15 \mathrm{~mL})$. The extract was dried over magnesium sulfate, and concentrated in vacuo to provide crude 2-chloro-4-substituted pyrimidines 4 that without purification were used for the reaction with $N$-methylpiperazine. Thus, a solution of crude compound 4 and $N$-methylpiperazine $(0.14 \mathrm{~mL}$, $1.2 \mathrm{mmol})$ in toluene $(4.0 \mathrm{~mL})$ was heated to $80^{\circ} \mathrm{C}$ until a TLC analysis on silica gel eluting with hexanes/ethyl ether (4:1) showed the absence of 4 (several hours). The mixture was cooled to room temperature, basified with sodium carbonate and extracted with ethyl ether $(3 \times 15 \mathrm{~mL})$. The extract was dried over magnesium sulfate, and concentrated under reduced pressure to provide crude compounds 5-12 that were purified by chromatography eluting with hexanes/ethyl ether/methanol (16:3:1). A single compound $4(\mathrm{R}=\mathrm{Ph}, \mathrm{Nu}=\mathrm{OMe}$ in Scheme 1$)$ was purified by chromatography in a similar manner.

2-Chloro-4-(2-methoxy-1-phenylethyl)pyrimidine $(4, \mathrm{R}=\mathrm{Ph}, \mathrm{Nu}=\mathrm{OMe})$. This compound was obtained as an oil in 25\%; ${ }^{1} \mathrm{H}-\mathrm{NMR}\left(\mathrm{CDCl}_{3}\right): \delta 8.47(\mathrm{~d}, J=5.2 \mathrm{~Hz}, 1 \mathrm{H}), 7.29(\mathrm{~m}, 4 \mathrm{H}), 7.26(\mathrm{~m}, 1 \mathrm{H})$, $7.12(\mathrm{~d}, J=5.2 \mathrm{~Hz}, 1 \mathrm{H}), 4.31(\mathrm{dd}, J=8.8,5.6 \mathrm{~Hz}, 1 \mathrm{H}), 4.20(\mathrm{t}, J=8.8 \mathrm{~Hz}, 1 \mathrm{H}), 3.88(\mathrm{dd}, J=8.8$, $5.6 \mathrm{~Hz}, 1 \mathrm{H}), 3.35(\mathrm{~s}, 3 \mathrm{H}) ;{ }^{13} \mathrm{C}-\mathrm{NMR}\left(\mathrm{CDCl}_{3}\right): \delta 173.6,161.3,159.3,138.4,128.9,128.3,127.6,119.5$, 74.5, 59.1, 53.2. High-resolution MS (ESI, positive ion mode): calcd. for $\mathrm{C}_{13} \mathrm{H}_{13}{ }^{35} \mathrm{ClN}_{2} \mathrm{O}\left(\mathrm{M}^{+}+1\right), \mathrm{m} / \mathrm{z}$ 249.0795; found $\mathrm{m} / \mathrm{z} 249.0784$.

N,N-Dimethyl-2(2-(4-methylpiperazino)pyrimidin-4-yl)ethanamine (5). This compound was obtained as a brown oil in 55\% yield; ${ }^{1} \mathrm{H}-\mathrm{NMR}\left(\mathrm{CDCl}_{3}\right): \delta 8.20(\mathrm{~d}, J=4.8 \mathrm{~Hz}, 1 \mathrm{H}), 6.41(\mathrm{~d}, J=4.8 \mathrm{~Hz}, 1 \mathrm{H})$, $3.86(\mathrm{t}, J=5.2 \mathrm{~Hz}, 4 \mathrm{H}), 2.77-2.74(\mathrm{~m}, 2 \mathrm{H}), 2.71-2.69(\mathrm{~m}, 2 \mathrm{H}), 2.48(\mathrm{t}, J=5.2 \mathrm{~Hz}, 4 \mathrm{H}), 2.35(\mathrm{~s}, 3 \mathrm{H})$, $2.31(\mathrm{~s}, 6 \mathrm{H}) ;{ }^{13} \mathrm{C}-\mathrm{NMR}\left(\mathrm{CDCl}_{3}\right): \delta 169.2,161.7,157.4,109.4,57.9,54.9,46.2,45.2,43.6,35.7$. Highresolution MS (ESI, positive ion mode): calcd. for $\mathrm{C}_{13} \mathrm{H}_{23} \mathrm{~N}_{5}\left(\mathrm{M}^{+}+1\right), \mathrm{m} / \mathrm{z}$ 250.2007; found $\mathrm{m} / \mathrm{z}$ 250.2032 .

$\mathrm{N}$-(2-(2-(4-Methylpiperazino)pyrimidin-4-yl)ethyl)butan-1-amine (6). This compound was obtained as an oil in 46\% yield; ${ }^{1} \mathrm{H}-\mathrm{NMR}\left(\mathrm{CDCl}_{3}\right): \delta 8.19(\mathrm{~d}, J=5.0 \mathrm{~Hz}, 1 \mathrm{H}), 6.38(\mathrm{~d}, J=5.0 \mathrm{~Hz}, 1 \mathrm{H}), 3.84(\mathrm{t}$, $J=5.0 \mathrm{~Hz}, 4 \mathrm{H}), 2.97(\mathrm{t}, J=6.6 \mathrm{~Hz}, 2 \mathrm{H}), 2.77(\mathrm{t}, J=6.6 \mathrm{~Hz}, 2 \mathrm{H}), 2.63(\mathrm{t}, J=7.2 \mathrm{~Hz}, 2 \mathrm{H}), 2.46(\mathrm{t}$, $J=4.8 \mathrm{~Hz}, 4 \mathrm{H}), 2.34(\mathrm{~s}, 3 \mathrm{H}), 1.49-1.43(\mathrm{~m}, 2 \mathrm{H}), 0.93-0.89(\mathrm{~m}, 2 \mathrm{H}), 0.91(\mathrm{t}, J=7.2 \mathrm{~Hz}, 3 \mathrm{H})$; ${ }^{13} \mathrm{C}-\mathrm{NMR}\left(\mathrm{CDCl}_{3}\right): \delta 169.4,161.7,157.3,157.1,109.3,54.9,49.5,48.1,46.2,43.6,37.7,20.5,14.0$. High-resolution MS (ESI, positive ion mode): calcd. for $\mathrm{C}_{15} \mathrm{H}_{26} \mathrm{~N}_{5}\left(\mathrm{M}^{+}+1\right), \mathrm{m} / \mathrm{z} 278.2340$; found $\mathrm{m} / \mathrm{z}$ 278.2345 . 
2-Methyl-N-(2-(2-(4-methylpiperazino)pyrimidin-4-yl)ethyl)propan-2-amine (7). This compound was obtained as an oil in 38\% yield; ${ }^{1} \mathrm{H}$ NMR $\left(\mathrm{CDCl}_{3}\right): \delta 8.20(\mathrm{~d}, J=4.8 \mathrm{~Hz}, 1 \mathrm{H}), 6.40(\mathrm{~d}, J=5.0 \mathrm{~Hz}, 1 \mathrm{H})$, $3.85(\mathrm{t}, J=3.2 \mathrm{~Hz}, 4 \mathrm{H}), 2.97-2.92(\mathrm{~m}, 2 \mathrm{H}), 2.79-2.75(\mathrm{~m}, 2 \mathrm{H}), 2.48(\mathrm{t}, J=5.0 \mathrm{~Hz}, 4 \mathrm{H}), 2.34(\mathrm{~s}, 3 \mathrm{H})$, $1.11(\mathrm{~s}, 9 \mathrm{H}) ;{ }^{13} \mathrm{C} \mathrm{NMR}\left(\mathrm{CDCl}_{3}\right): \delta 169.5,161.6,157.4,109.4,55.0,50.2,46.2,43.6,40.8,38.4,29.0$. High-resolution MS (ESI, positive ion mode): calcd. for $\mathrm{C}_{15} \mathrm{H}_{26} \mathrm{~N}_{5}\left(\mathrm{M}^{+}+1\right), \mathrm{m} / \mathrm{z} 278.2345$; found $\mathrm{m} / \mathrm{z}$ 278.23.

N-Benzyl-2-(2-(4-methylpiperazino)pyrimidin-4-yl)ethanamine tetrahydrobromide (8). This salt was obtained in $62 \%$ yield; m.p. $110-112{ }^{\circ} \mathrm{C} ;{ }^{1} \mathrm{H}-\mathrm{NMR}$ (DMSO- $\left.d_{6}\right): \delta 10.0$ (bs, $\left.1 \mathrm{H}\right), 8.96$ (bs, $\left.1 \mathrm{H}\right), 8.37(\mathrm{~d}$, $J=4.8 \mathrm{~Hz}, 1 \mathrm{H}), 7.58-7.55(\mathrm{~m}, 2 \mathrm{H}), 7.49-7.42(\mathrm{~m}, 3 \mathrm{H}), 6.73(\mathrm{~d}, J=5.4 \mathrm{~Hz}, 1 \mathrm{H}), 4.68(\mathrm{~d}, J=14.4 \mathrm{~Hz}$, 2H), $4.24(\mathrm{t}, J=5.4 \mathrm{~Hz}, 2 \mathrm{H}), 3.55-3.50(\mathrm{~m}, 2 \mathrm{H}), 3.39-3.29(\mathrm{~m}, 4 \mathrm{H}), 3.06(\mathrm{t}, J=7.6 \mathrm{~Hz}, 4 \mathrm{H}), 2.86(\mathrm{bs}$, $3 \mathrm{H}) ;{ }^{13} \mathrm{C}-\mathrm{NMR}\left(\mathrm{CDCl}_{3}\right): \delta 171.3,163.2,158.3,143.2,128.3,128.1,125.9,110.2,55.3,54.2,46.3$, 44.0, 39.3, 34.2. MS (ESI) $\mathrm{m} / \mathrm{z} 312\left(\mathrm{M}^{+}+\mathrm{H}\right)$. Anal. Calcd for $\mathrm{C}_{18} \mathrm{H}_{25} \mathrm{~N}_{5} \cdot 4 \mathrm{HBr} \cdot \mathrm{H}_{2} \mathrm{O}: \mathrm{C}, 33.10 ; \mathrm{H}, 4.78$; N, 10.72. Found: C, 33.43; H, 5.10; N, 10.66 .

4-(2-Methoxyethyl)-2-4-methylpiperazino)pyrimidine trihydrobromide (9). The free base was obtained as an oil in 55\% yield; ${ }^{1} \mathrm{H}-\mathrm{NMR}$ for the free base $\left(\mathrm{CDCl}_{3}\right): \delta 8.22(\mathrm{~d}, J=5.0 \mathrm{~Hz}, 1 \mathrm{H}), 6.44(\mathrm{~d}$, $J=5.0 \mathrm{~Hz}, 1 \mathrm{H}), 3.87(\mathrm{t}, J=5.0 \mathrm{~Hz}, 4 \mathrm{H}), 3.77(\mathrm{t}, J=6.8 \mathrm{~Hz}, 2 \mathrm{H}), 3.37(\mathrm{~s}, 3 \mathrm{H}), 2.85$ (t, $J=6.8 \mathrm{~Hz}$, $2 \mathrm{H}), 2.48(\mathrm{t}, J=4.8 \mathrm{~Hz}, 4 \mathrm{H}), 2.36(\mathrm{~s}, 3 \mathrm{H}) ;{ }^{13} \mathrm{C}-\mathrm{NMR}\left(\mathrm{CDCl}_{3}\right): \delta 168.1,161.7,157.4,109.4,58.7,55.0$, 46.2, 43.6, 38.0, 29.7. High-resolution MS for the free base (ESI, positive ion mode): calcd. for $\mathrm{C}_{12} \mathrm{H}_{20}$ $\mathrm{N}_{4} \mathrm{O}\left(\mathrm{M}^{+}+1\right), \mathrm{m} / \mathrm{z} 237.1705$; found $\mathrm{m} / \mathrm{z}$ 237.1715. Anal. calcd for: calcd for $\mathrm{C}_{12} \mathrm{H}_{20} \mathrm{~N}_{4} \mathrm{O} \cdot 3 \mathrm{HBr}$ : C, 30.09; H, 4.24; N, 11.70. Found: C, 29.86; H, 4.34; N, 12.12.

4-(2-(Ethylthio)ethyl)-2-(4-methylpiperazino)pyrimidine (10). This compound was obtained as an oil in 25\% yield; ${ }^{1} \mathrm{H}-\mathrm{NMR}\left(\mathrm{CDCl}_{3}\right): \delta 8.22(\mathrm{~d}, J=5.0 \mathrm{~Hz}, 1 \mathrm{H}), 6.41(\mathrm{~d}, J=5.0 \mathrm{~Hz}, 1 \mathrm{H}), 3.87(\mathrm{t}, J=4.8 \mathrm{~Hz}$, 4H), 2.92-2.90 (m, 2H), 2.88-2.86 (m, 2H), 2.60 (q, $J=7.4 \mathrm{~Hz}, 2 \mathrm{H}), 2.49$ (t, $J=4.8 \mathrm{~Hz}, 4 \mathrm{H}), 2.36$ (s, $3 \mathrm{H}), 1.29(\mathrm{t}, J=7.4 \mathrm{~Hz}, 3 \mathrm{H}) ;{ }^{13} \mathrm{C}-\mathrm{NMR}\left(\mathrm{CDCl}_{3}\right): \delta 168.9,161.7,157.4,109.1,55.0,46.2,43.6,38.0$, 29.8, 26.0, 14.7. High-resolution MS (ESI, positive ion mode): calcd. for $\mathrm{C}_{13} \mathrm{H}_{22} \mathrm{~N}_{4} \mathrm{~S}\left(\mathrm{M}^{+}+1\right), \mathrm{m} / \mathrm{z}$ 267.1632; found $\mathrm{m} / \mathrm{z} 267.1643$.

N-Benzyl-2-(2-(4-methylpiperazino)pyrimidin-4-yl)-2-phenylethanamine (11). This compound was obtained as an oil in 57\% yield; ${ }^{1} \mathrm{H}-\mathrm{NMR}\left(\mathrm{CDCl}_{3}\right) \delta 8.13(\mathrm{~d}, J=5.1 \mathrm{~Hz}, 1 \mathrm{H}), 7.25(\mathrm{~m}, 10 \mathrm{H}), 6.29$ $(\mathrm{d}, J=5.1 \mathrm{~Hz}, 1 \mathrm{H}), 4.11(\mathrm{dd}, J=6.4,8.4 \mathrm{~Hz}, 1 \mathrm{H}), 3.82(\mathrm{~m}, 6 \mathrm{H}), 3.49(\mathrm{dd}, J=8.4,11.8 \mathrm{~Hz}, 1 \mathrm{H}), 3.07$ $(\mathrm{dd}, J=6.4,11.8 \mathrm{~Hz}, 1 \mathrm{H}), 2.45(\mathrm{~m}, 4 \mathrm{H}), 2.34(\mathrm{~s}, 3 \mathrm{H}) ;{ }^{13} \mathrm{C}-\mathrm{NMR}\left(\mathrm{CDCl}_{3}\right) \delta 170.8,161.6,157.7,141.1$, 140.3 , 128.6, 128.4, 128.3, 128.1, 127.0, 126.9, 109.7, 55.0, 53.9, 53.1, 53.0, 46.3, 43.7. High resolution MS (ESI, positive ion mode): calcd. for $\mathrm{C}_{24} \mathrm{H}_{29} \mathrm{~N}_{5},\left(\mathrm{M}^{+}+1\right), \mathrm{m} / \mathrm{z} 388.2501$, found $\mathrm{m} / \mathrm{z}$ 388.2491 .

4-(2-Ethylthio)-1-phenylethyl)-2-(4-methylpiperazino)pyrimidine (12). This compound was obtained as an oil in $37 \%$ yield; ${ }^{1} \mathrm{H}-\mathrm{NMR}\left(\mathrm{CDCl}_{3}\right) \delta 8.15(\mathrm{~d}, J=5.2 \mathrm{~Hz}, 1 \mathrm{H}), 7.29(\mathrm{~m}, 5 \mathrm{H}), 6.34(\mathrm{~d}, J=5.2 \mathrm{~Hz}$, $1 \mathrm{H}), 4.01(\mathrm{~m}, 1 \mathrm{H}), 3.88(\mathrm{~m}, 4 \mathrm{H}), 3.48(\mathrm{~m}, 1 \mathrm{H}), 3.06(\mathrm{~m}, 1 \mathrm{H}), 2.48(\mathrm{~m}, 6 \mathrm{H}), 2.35(\mathrm{~s}, 3 \mathrm{H}), 1.22(\mathrm{t}$, $J=7.2 \mathrm{~Hz}, 3 \mathrm{H}) ;{ }^{13} \mathrm{C}-\mathrm{NMR}\left(\mathrm{CDCl}_{3}\right): \delta 170.7,161.6,157.7,141.7,128.5,128.1,127.1,109.4,55.0$, 
53.8, 46.3, 43.7, 36.1, 26.7, 14.8. High-resolution MS (ESI, positive ion mode): calcd. for $\mathrm{C}_{19} \mathrm{H}_{26} \mathrm{~N}_{4} \mathrm{~S}$ $\left(\mathrm{M}^{+}+1\right), \mathrm{m} / \mathrm{z} 343.1956$; found $\mathrm{m} / \mathrm{z} 343.1947$.

$\mathrm{N}, \mathrm{N}$-bis-\{[2-(4-Methylpiperazino)pyrimidin-4-yl]ethyl\}ethanamine $\quad(\mathbf{1 4}) . \quad$ Ethylamine $\quad(70 \% \quad$ in tetrahydrofuran $(0.04 \mathrm{~mL}, 0.55 \mathrm{mmol})$ was added to a solution of 2-chloro-4-vinylpyrimidine (2, $0.14 \mathrm{~g}, 1.0 \mathrm{mmol})$ in toluene $(2.0 \mathrm{~mL})$. The mixture was stirred for $48 \mathrm{~h}$ at $23{ }^{\circ} \mathrm{C}$ and then heated to $110^{\circ} \mathrm{C}$ for $2 \mathrm{~h}$ after which time a TLC analysis showed the absence of 2 . The mixture was cooled to room temperature, extracted with ethyl ether $(2 \times 15 \mathrm{~mL})$, dried over magnesium sulfate and concentrated on a rotary evaporator. The residue was purified by chromatography eluting with hexanes/ethyl ether/methanol $(8: 1: 1)$ to provide $\mathbf{1 4}$ as a yellow oil in $38 \%$ yield $(88 \mathrm{mg})$; ${ }^{1} \mathrm{H}-\mathrm{NMR}$ $\left(\mathrm{CDCl}_{3}\right): \delta 8.12(\mathrm{~d}, J=4.8 \mathrm{~Hz}, 2 \mathrm{H}), 6.37(\mathrm{~d}, J=4.8 \mathrm{~Hz}, 2 \mathrm{H}), 3.87(\mathrm{t}, J=4.6 \mathrm{~Hz}, 8 \mathrm{H}), 2.91(\mathrm{q}$, $J=8.0 \mathrm{~Hz}, 4 \mathrm{H}), 2.73(\mathrm{q}, J=8.0 \mathrm{~Hz}, 4 \mathrm{H}), 2.64(\mathrm{q}, J=7.2 \mathrm{~Hz}, 2 \mathrm{H}), 2.47(\mathrm{t}, J=4.6 \mathrm{~Hz}, 8 \mathrm{H}), 2.35$ (s, $6 \mathrm{H}), 1.04(\mathrm{t}, J=7.2 \mathrm{~Hz}, 3 \mathrm{H}) ;{ }^{13} \mathrm{C}-\mathrm{NMR}\left(\mathrm{CDCl}_{3}\right): \delta 169.7,162.3,157.2,109.4,55.0,51.9,47.4,46.3$, 43.6, 35.3, 12.1. High-resolution MS (ESI, positive ion mode): calcd. for $\mathrm{C}_{24} \mathrm{H}_{39} \mathrm{~N}_{9}\left(\mathrm{M}^{+}+1\right), \mathrm{m} / \mathrm{z}$ 454.3405; found $\mathrm{m} / \mathrm{z} 454.3407$.

\subsection{General procedure for pyrimidines $\mathbf{1 5 - 1 7}$}

Methylmagnesium bromide ( $3 \mathrm{M}$ in ether, $0.34 \mathrm{~mL}, 1 \mathrm{mmol}$ ) or phenylmagnesium bromide $(1 \mathrm{M}$ in tetrahydrofuran, $1.0 \mathrm{~mL}, 1.0 \mathrm{mmol}$ ) was added to cuprous iodide $(94 \mathrm{mg}, 0.5 \mathrm{mmol})$ in tetrahydrofuran $(2 \mathrm{~mL})$ at $-50{ }^{\circ} \mathrm{C}$. The mixture was stirred for $10 \mathrm{~min}$ at $-50{ }^{\circ} \mathrm{C}$ and then treated dropwise with a solution of compound $2(100 \mathrm{mg}, 0.7 \mathrm{mmol}$ or $3(160 \mathrm{mg}, 0.7 \mathrm{mmol})$ in tetrahydrofuran $(3 \mathrm{~mL})$. The mixture was stirred at $-40{ }^{\circ} \mathrm{C}$ for $2 \mathrm{~h}$, then quenched with aqueous solution of ammonium chloride $(2 \mathrm{M}, 2.0 \mathrm{~mL})$ and extracted with ethyl acetate $(3 \times 15 \mathrm{~mL})$. The extract was dried over magnesium sulfate and concentrated on a rotary evaporator. A solution of the residue and $N$-methylpiperazine $(0.12 \mathrm{~mL}, 1.0 \mathrm{mmol})$ in toluene $(3.0 \mathrm{~mL})$ was heated to $120^{\circ} \mathrm{C}$ until a TLC analysis showed the absence of the Grignard adduct (several hours). The mixture was cooled to room temperature, basified with sodium carbonate, and extracted with ethyl ether $(3 \times 10 \mathrm{~mL})$. The extract was dried over magnesium sulfate and concentrated on a rotary evaporator. The residue was subjected to chromatography eluting with hexanes/ether/methanol (16:3:1) to provide analytically pure products 15-17.

2-(4-Methylpiperazino)-4-propylpyrimidine (15). This compound was obtained as an oil in 56\% yield; ${ }^{1} \mathrm{H}-\mathrm{NMR}\left(\mathrm{CDCl}_{3}\right): \delta 8.22(\mathrm{~d}, J=5.2 \mathrm{~Hz}, 1 \mathrm{H}), 6.46(\mathrm{~d}, J=5.2 \mathrm{~Hz}, 1 \mathrm{H}), 4.67(\mathrm{~d}, J=14.0 \mathrm{~Hz}, 2 \mathrm{H}), 3.96$ $(\mathrm{t}, J=14.0 \mathrm{~Hz}, 2 \mathrm{H}), 3.36-3.25(\mathrm{~m}, 7 \mathrm{H}), 2.56(\mathrm{t}, J=7.6 \mathrm{~Hz}, 2 \mathrm{H}), 1.75-1.70(\mathrm{~m}, 2 \mathrm{H}), 0.97(\mathrm{t}$, $J=7.6 \mathrm{~Hz}, 3 \mathrm{H}) ;{ }^{13} \mathrm{C}-\mathrm{NMR}\left(\mathrm{CDCl}_{3}\right): \delta 170.2,160.3,157.3,110.0,65.8,60.5,39.8,38.6,21.6,13.8$. High-resolution MS (ESI, positive ion mode): calcd. for $\mathrm{C}_{12} \mathrm{H}_{20} \mathrm{~N}_{4}\left(\mathrm{M}^{+}+1\right), \mathrm{m} / \mathrm{z} 220.1700$; found $\mathrm{m} / \mathrm{z}$ 220.1706 .

2-(4-Methylpiperazino)-4-(2-phenylethyl)pyrimidine (16). This compound was obtained as an oil in 77\% yield; ${ }^{1} \mathrm{H}-\mathrm{NMR}\left(\mathrm{CDCl}_{3}\right): \delta 8.19(\mathrm{~d}, J=5.0 \mathrm{~Hz}, 1 \mathrm{H}), 7.31(\mathrm{t}, J=7.2 \mathrm{~Hz}, 2 \mathrm{H}), 7.23-7.21(\mathrm{~m}, 3 \mathrm{H})$, $6.35(\mathrm{~d}, J=5.0 \mathrm{~Hz}, 1 \mathrm{H}), 3.88(\mathrm{t}, J=4.8 \mathrm{~Hz}, 4 \mathrm{H}), 3.04(\mathrm{q}, J=7.2 \mathrm{~Hz}, 2 \mathrm{H}), 2.90(\mathrm{q}, J=7.2 \mathrm{~Hz}, 2 \mathrm{H})$, 
$2.49(\mathrm{t}, J=4.8 \mathrm{~Hz}, 4 \mathrm{H}), 2.37(\mathrm{~s}, 3 \mathrm{H}) ;{ }^{13} \mathrm{C}-\mathrm{NMR}\left(\mathrm{CDCl}_{3}\right): \delta 170.0,161.7,157.3,141.4,128.4,128.3$, 125.9, 109.0, 55.0, 46.3, 43.6, 39.4, 34.2. High-resolution MS (ESI, positive ion mode): calcd. for $\mathrm{C}_{17} \mathrm{H}_{22} \mathrm{~N}_{4}\left(\mathrm{M}^{+}+1\right), \mathrm{m} / \mathrm{z} 283.1931$; found $\mathrm{m} / \mathrm{z} 283.1923$.

2-(4-Methylpiperazino)-4-(1-phenylpropyl)pyrimidine (17). This compound was obtained as an oil in $40 \%$ yield; ${ }^{1} \mathrm{H}-\mathrm{NMR}\left(\mathrm{CDCl}_{3}\right) \delta 8.13(\mathrm{~d}, J=5.2 \mathrm{~Hz}, 1 \mathrm{H}), 7.30(\mathrm{~m}, 4 \mathrm{H}), 7.20(\mathrm{~m}, 1 \mathrm{H}), 6.32(\mathrm{~d}$, $J=5.2 \mathrm{~Hz}, 1 \mathrm{H}), 3.86(\mathrm{t}, J=5.2 \mathrm{~Hz}, 4 \mathrm{H}), 3.66(\mathrm{t}, J=7.6 \mathrm{~Hz}, 1 \mathrm{H}), 2.46(\mathrm{t}, J=5.2 \mathrm{~Hz}, 4 \mathrm{H}), 2.34(\mathrm{~s}$, $3 \mathrm{H}), 2.23(\mathrm{~m}, 1 \mathrm{H}), 1.99(\mathrm{~m}, 1 \mathrm{H}), 0.88(\mathrm{t}, J=7.6 \mathrm{~Hz}, 3 \mathrm{H}) ;{ }^{13} \mathrm{C}-\mathrm{NMR}\left(\mathrm{CDCl}_{3}\right) \delta 172.5,161.7,157.4$, 142.8, 128.3, 128.2, 126.5, 109.0, 55.4, 55.0, 46.3, 43.7, 27.5, 12.5. High-resolution MS (ESI, positive ion mode): calcd. for $\mathrm{C}_{18} \mathrm{H}_{24} \mathrm{~N}_{4}(\mathrm{M}+1)^{+}, \mathrm{m} / \mathrm{z} 297.2079$; found $\mathrm{m} / \mathrm{z} 297.2089$.

\subsection{General procedure for quinazolines $\mathbf{2 0 - 2 6}$}

Synthesis of 2-chloro-4-vinylquinazoline (19) has been reported by us recently [7]. To a solution of $19(190 \mathrm{mg}, 1.0 \mathrm{mmol})$ in toluene $(3.0 \mathrm{~mL})$ was added the corresponding nucleophile (amine, MeONa, EtSNa, PhSNa, $1.0 \mathrm{mmol}$ ). The mixture was stirred at room temperature overnight or at $90{ }^{\circ} \mathrm{C}$ for $2 \mathrm{~h}$, then treated at room temperature with an aqueous solution of ammonium chloride $(2 \mathrm{M}, 2 \mathrm{~mL})$, and extracted with ether $(3 \times 15 \mathrm{~mL})$. The extract was dried over magnesium sulfate and concentrated under reduced pressure to provide crude 4-substituted 2-chloroquinazoline that without purification was used for the reaction with $\mathrm{N}$-methylpiperazine. Thus, a solution of the residue after concentration and $N$-methylpiperazine $(0.30 \mathrm{~mL}, 2.7 \mathrm{mmol})$ in toluene $(3.0 \mathrm{~mL})$ was heated to $80{ }^{\circ} \mathrm{C}$ until a TLC analysis on silica gel eluting with hexanes/ether/methanol (16:3:1) showed the absence of the intermediate product (several hours). The solution was cooled to room temperature, basified with sodium carbonate, and extracted with ethyl ether $(3 \times 15 \mathrm{~mL})$. The extract was dried over magnesium sulfate and concentrated on a rotary evaporator to provide crude compounds 20-26. These products were purified by chromatography eluting with hexanes/ether/methanol (5:4:1).

N,N-Dimethyl-2-(2-(4-methylpiperazino)quinazolin-4-yl)ethanamine hydrobromide (20). The free base was obtained as a yellow oil in $27 \%$ yield; ${ }^{1} \mathrm{H}-\mathrm{NMR}$ for the free base $\left(\mathrm{CDCl}_{3}\right): \delta 7.86(\mathrm{~d}, \mathrm{~J}=8.0 \mathrm{~Hz}$, 1H), 7.60-7.57 (m, 2H), 7.18 (t, $J=7.2 \mathrm{~Hz}, 1 \mathrm{H}), 3.99$ (t, $J=5.0 \mathrm{~Hz}, 4 \mathrm{H}), 3.29$ (t, $J=7.2 \mathrm{~Hz}, 2 \mathrm{H}), 2.85$ $(\mathrm{t}, J=8.0 \mathrm{~Hz}, 2 \mathrm{H}), 2.51(\mathrm{t}, J=5.0 \mathrm{~Hz}, 4 \mathrm{H}), 2.36(\mathrm{~s}, 6 \mathrm{H}), 2.35(\mathrm{~s}, 3 \mathrm{H}) ;{ }^{13} \mathrm{C}-\mathrm{NMR}$ for the free base $\left(\mathrm{CDCl}_{3}\right): \delta 170.1,158.4,152.3,133.3,126.4,124.6,122.1,118.6,57.6,55.1,46.3,45.5,43.8,32.4$. High-resolution MS (ESI, positive ion mode): calcd. for $\mathrm{C}_{17} \mathrm{H}_{25} \mathrm{~N}_{5}\left(\mathrm{M}^{+}+1\right), \mathrm{m} / \mathrm{z} 300.2175$; found $\mathrm{m} / \mathrm{z}$ 300.2188. A hydrobromide salt: m.p. $100-106{ }^{\circ} \mathrm{C}$ (dec.). Anal. Calcd. for $\mathrm{C}_{17} \mathrm{H}_{25} \mathrm{~N}_{5} \cdot 3.5 \mathrm{HBr} \cdot \mathrm{H}_{2} \mathrm{O}: \mathrm{C}$, 34.00; H, 5.12; N, 11.66. Found: C, 33.74; H, 5.40; N, 11.26.

$N$-\{2-[2-(4-Methylpiperazino)quinazolin-4-yl]ethyl\}butanamine trihydrobromide (21). The free base was obtained as a brown oil in $31 \%$ yield; ${ }^{1} \mathrm{H}-\mathrm{NMR}$ for the free base $\left(\mathrm{CDCl}_{3}\right): \delta 7.83(\mathrm{~d}, \mathrm{~J}=8.0 \mathrm{~Hz}$, $1 \mathrm{H}), 7.62-7.53(\mathrm{~m}, 2 \mathrm{H}), 7.14(\mathrm{~d}, J=8.0 \mathrm{~Hz}, 1 \mathrm{H}), 3.96(\mathrm{~s}, 4 \mathrm{H}), 3.49(\mathrm{t}, J=6.6 \mathrm{~Hz}, 2 \mathrm{H}), 3.30$ (t, $J=6.6 \mathrm{~Hz}, 2 \mathrm{H}), 2.81(\mathrm{t}, J=7.2 \mathrm{~Hz}, 2 \mathrm{H}), 2.51(\mathrm{t}, J=4.8 \mathrm{~Hz}, 4 \mathrm{H}), 2.36(\mathrm{~s}, 3 \mathrm{H}), 1.64-1.59(\mathrm{~m}, 3 \mathrm{H})$, $1.40-1.32(\mathrm{~m}, 2 \mathrm{H}), 0.90(\mathrm{t}, J=7.2 \mathrm{~Hz}, 3 \mathrm{H}) ;{ }^{13} \mathrm{C}-\mathrm{NMR}$ for the free base $\left(\mathrm{CDCl}_{3}\right): \delta 169.1,158.3,152.5$, 133.8, 126.6, 124.7, 122.6, 118.6, 55.2, 49.3, 46.8, 46.4, 44.1, 32.5, 31.2, 20.6, 14.0; High-resolution 
MS (ESI, positive ion mode): calcd. for $\mathrm{C}_{19} \mathrm{H}_{29} \mathrm{~N}_{5}\left(\mathrm{M}^{+}+1\right), \mathrm{m} / \mathrm{z} 328.2503$; found $\mathrm{m} / \mathrm{z}$ 328.2501. A hydrobromide salt: m.p. $153-155{ }^{\circ} \mathrm{C}$. Anal. Calcd. for $\mathrm{C}_{19} \mathrm{H}_{29} \mathrm{~N}_{5} \cdot 3 \mathrm{HBr} \cdot 3 \mathrm{H}_{2} \mathrm{O}: \mathrm{C}, 36.04 ; \mathrm{H}, 6.21 ; \mathrm{N}$, 11.06. Found: C, 35.89; H, 6.00; N, 11.28.

N-Benzyl-2-(2-(4-methylpiperazino)quinazolin-4-yl)ethanamine trihydrobromide (22). The free base was obtained as a yellow oil in 50\% yield; ${ }^{1} \mathrm{H}-\mathrm{NMR}$ for the free base $\left(\mathrm{CDCl}_{3}\right): \delta 7.84(\mathrm{~d}, \mathrm{~J}=8.0 \mathrm{~Hz}$, 1H), 7.64-7.56 (m, 2H), 7.33 (d, J=4.4 Hz, 4H), 7.28-7.25 (m, 1H), 7.18 (t, J=8.0 Hz, 1H), $3.96(\mathrm{~d}$, $J=4.6 \mathrm{~Hz}, 4 \mathrm{H}), 3.88(\mathrm{~s}, 2 \mathrm{H}), 3.38(\mathrm{t}, J=6.2 \mathrm{~Hz}, 2 \mathrm{H}), 3.22(\mathrm{t}, J=6.2 \mathrm{~Hz}, 2 \mathrm{H}), 2.49$ (t, $J=4.6 \mathrm{~Hz}, 4 \mathrm{H})$, $2.36(\mathrm{~s}, 3 \mathrm{H}), 2.26(\mathrm{bs}, 1 \mathrm{H}) ;{ }^{13} \mathrm{C}-\mathrm{NMR}$ for the free base $\left(\mathrm{CDCl}_{3}\right): \delta 170.1,158.4,152.4,140.2,133.6$, 128.6, 128.3, 127.2, 126.5, 124.7, 122.4, 118.8, 55.2, 54.2, 46.7, 46.4, 44.0, 33.7. High-resolution MS (ESI, positive ion mode): calcd. for $\mathrm{C}_{22} \mathrm{H}_{27} \mathrm{~N}_{5}\left(\mathrm{M}^{+}+1\right), \mathrm{m} / \mathrm{z} 362.2354$; found $\mathrm{m} / \mathrm{z}$ 362.2345. A hydrobromide salt: m.p. $160-162{ }^{\circ} \mathrm{C}$. Anal. Calcd. for $\mathrm{C}_{22} \mathrm{H}_{27} \mathrm{~N}_{5} \cdot 3 \mathrm{HBr}$ : C, 43.73; H, 5.00; N, 11.59 . Found: C, 43.88; H, 5.48; N, 11.39.

2-(4-Methylpiperazino)-4-(2-(4-phenylpiperazino)ethyl)quinazoline tetrahydrobromide (23). The free base was obtained as a brown oil in $23 \%$ yield; ${ }^{1} \mathrm{H}$-NMR for the free base $\left(\mathrm{CDCl}_{3}\right): \delta 7.90(\mathrm{~d}$, $J=8.0 \mathrm{~Hz}, 1 \mathrm{H}), 7.66-7.58(\mathrm{~m}, 2 \mathrm{H}), 7.31-7.27(\mathrm{~m}, 2 \mathrm{H}), 7.21(\mathrm{t}, J=8.0 \mathrm{~Hz}, 1 \mathrm{H}), 6.97(\mathrm{~d}, J=8.0 \mathrm{~Hz}$, $2 \mathrm{H}), 6.88(\mathrm{t}, J=7.2 \mathrm{~Hz}, 1 \mathrm{H}), 4.03(\mathrm{~s}, 4 \mathrm{H}), 3.39(\mathrm{t}, J=7.8 \mathrm{~Hz}, 2 \mathrm{H}), 3.27(\mathrm{t}, J=4.8 \mathrm{~Hz}, 4 \mathrm{H}), 3.02$ $(\mathrm{t}, J=7.8 \mathrm{~Hz}, 2 \mathrm{H}), 2.79(\mathrm{t}, J=4.8 \mathrm{~Hz}, 4 \mathrm{H}), 2.55(\mathrm{t}, J=6.0 \mathrm{~Hz}, 4 \mathrm{H}), 2.39(\mathrm{~s}, 3 \mathrm{H}) ;{ }^{13} \mathrm{C}-\mathrm{NMR}$ for the free base $\left(\mathrm{CDCl}_{3}\right): \delta 170.2,159.3,152.5,151.4,133.6,129.3,126.7,124.8,122.5,119.9,118.8$, 116.3, 56.6, 55.2, 53.4, 49.3, 46.3, 43.9, 31.9. High-resolution MS (ESI, positive ion mode): calcd. for $\mathrm{C}_{25} \mathrm{H}_{32} \mathrm{~N}_{6}\left(\mathrm{M}^{+}+1\right), \mathrm{m} / \mathrm{z}$ 417.2751; found $\mathrm{m} / \mathrm{z}$ 417.2767. A hydrobromide salt: m.p. $190-194{ }^{\circ} \mathrm{C}$ (dec.). Anal. Calcd. for $\mathrm{C}_{25} \mathrm{H}_{32} \mathrm{~N}_{6} \cdot 4 \mathrm{HBr} \cdot 2 \mathrm{H}_{2} \mathrm{O}: \mathrm{C}, 38.68 ; \mathrm{H}, 5.19 ; \mathrm{N}, 10.83$. Found: C, 38.78; H, 5.51; N, 10.46 .

4-(2-Methoxyethyl)-2-(4-methylpiperazino)quinazoline (24). This compound was obtained as a yellow solid in 27\% yield; m.p. $35-37{ }^{\circ} \mathrm{C}$; ${ }^{1} \mathrm{H}-\mathrm{NMR}\left(\mathrm{CDCl}_{3}\right): \delta 7.86(\mathrm{~d}, J=8.0 \mathrm{~Hz}, 1 \mathrm{H}), 7.61-7.55(\mathrm{~m}, 2 \mathrm{H})$, $7.18(\mathrm{t}, J=8.0 \mathrm{~Hz}, 1 \mathrm{H}), 3.99(\mathrm{t}, J=4.8 \mathrm{~Hz}, 4 \mathrm{H}), 3.94(\mathrm{t}, J=7.2 \mathrm{~Hz}, 2 \mathrm{H}), 3.41-3.38(\mathrm{~m}, 5 \mathrm{H}), 2.51$ $(\mathrm{t}, J=4.8 \mathrm{~Hz}, 4 \mathrm{H}), 2.35(\mathrm{~s}, 3 \mathrm{H}) ;{ }^{13} \mathrm{C}-\mathrm{NMR}\left(\mathrm{CDCl}_{3}\right): \delta 168.9,158.4,152.3,133.4,126.4,124.7,122.2$, $118.8,70.5,58.8,55.1,46.3,43.8,34.1$. High-resolution MS (ESI, positive ion mode): calcd. for $\mathrm{C}_{16} \mathrm{H}_{22} \mathrm{~N}_{4} \mathrm{O}\left(\mathrm{M}^{+}+1\right), \mathrm{m} / \mathrm{z}$ 287.1874; Found $\mathrm{m} / \mathrm{z}$ 287.1872. Anal. Calcd. for $\mathrm{C}_{16} \mathrm{H}_{22} \mathrm{~N}_{4} \mathrm{O}: \mathrm{C}, 67.11 ; \mathrm{H}$, 7.74; N, 19.56. Found: C, 67.33; H, 8.05; N, 19.22.

4-(2-(Ethylthio)ethyl)-2-(4-methylpiperazino)quinazoline (25). This compound was obtained as a yellow solid in $35 \%$ yield; m.p. $38-40{ }^{\circ} \mathrm{C} ;{ }^{1} \mathrm{H}-\mathrm{NMR}\left(\mathrm{CDCl}_{3}\right): \delta 7.83(\mathrm{~d}, J=8.0 \mathrm{~Hz}, 1 \mathrm{H}), 7.63-7.55(\mathrm{~m}$, 2H), $7.18(\mathrm{t}, J=7.0 \mathrm{~Hz}, 1 \mathrm{H}), 4.00(\mathrm{t}, J=4.8 \mathrm{~Hz}, 4 \mathrm{H}), 3.41(\mathrm{t}, J=7.0 \mathrm{~Hz}, 2 \mathrm{H}), 3.07(\mathrm{t}, J=8.0 \mathrm{~Hz}, 2 \mathrm{H})$, $2.63(\mathrm{q}, J=7.0 \mathrm{~Hz}, 2 \mathrm{H}), 2.51(\mathrm{t}, J=4.8 \mathrm{~Hz}, 4 \mathrm{H}), 2.36(\mathrm{~s}, 3 \mathrm{H}), 1.30(\mathrm{t}, J=8.0 \mathrm{~Hz}, 3 \mathrm{H}) ;{ }^{13} \mathrm{C}-\mathrm{NMR}$ $\left(\mathrm{CDCl}_{3}\right): \delta 169.8,158.6,152.5,133.6,126.6,124.6,122.4,118.6,55.3,46.5,44.0,34.6,29.3,26.4$, 15.0. High-resolution MS (ESI, positive ion mode): calcd. for $\mathrm{C}_{17} \mathrm{H}_{24} \mathrm{~N}_{4} \mathrm{~S}\left(\mathrm{M}^{+}+1\right), \mathrm{m} / \mathrm{z} 317.1789$; Found $\mathrm{m} / \mathrm{z}$ 317.1800; Anal. Calcd. for $\mathrm{C}_{17} \mathrm{H}_{24} \mathrm{~N}_{4} \mathrm{~S}$ : C, 64.52; H, 7.64; N, 17.70. Found: C, 64.71; H, $7.81 ; \mathrm{N}, 17.30$. 
2-(4-Methylpiperazino)-4-(2-(phenylthio)ethyl)quinazoline dihydrobromide (26). This compound was obtained as a yellow oil in 50\% yield; ${ }^{1} \mathrm{H}-\mathrm{NMR}$ for the free base $\left(\mathrm{CDCl}_{3}\right): \delta 7.71(\mathrm{~d}, J=8.4 \mathrm{~Hz}, 1 \mathrm{H})$, $7.62-7.54(\mathrm{~m}, 2 \mathrm{H}), 7.40(\mathrm{~d}, J=7.6 \mathrm{~Hz}, 2 \mathrm{H}), 7.30(\mathrm{t}, J=7.6 \mathrm{~Hz}, 2 \mathrm{H}), 7.21$ (d, $J=7.2 \mathrm{~Hz}, 1 \mathrm{H}), 7.14$ (t, $J=6.8 \mathrm{~Hz}, 1 \mathrm{H}), 4.00(\mathrm{~s}, 4 \mathrm{H}), 3.51-3.43(\mathrm{~m}, 4 \mathrm{H}), 2.52(\mathrm{t}, J=5.2 \mathrm{~Hz}, 4 \mathrm{H}), 2.37(\mathrm{~s}, 3 \mathrm{H}) ;{ }^{13} \mathrm{C}-\mathrm{NMR}$ for the free base $\left(\mathrm{CDCl}_{3}\right): \delta 169.4,158.5,152.5,136.4,133.7,129.6,129.2,126.6,126.4,124.5,122.4$, 118.6, 55.3, 46.4, 44.0, 33.9, 31.2. High-resolution MS (ESI, positive ion mode): calcd. for $\mathrm{C}_{21} \mathrm{H}_{24} \mathrm{~N}_{4} \mathrm{~S}$ $\left(\mathrm{M}^{+}+1\right), \mathrm{m} / \mathrm{z}$ 365.1791; Found $\mathrm{m} / \mathrm{z}$ 365.1800. A hydrobromide salt: m.p. 135-137 ${ }^{\circ} \mathrm{C}$. Anal. Calcd. for $\mathrm{C}_{21} \mathrm{H}_{24} \mathrm{~N}_{4} \mathrm{~S} \cdot 2 \mathrm{HBr} \cdot \mathrm{H}_{2} \mathrm{O}: \mathrm{C}, 46.34 ; \mathrm{H}, 5.18 ; \mathrm{N}, 10.28$. Found: C, 46.14; H, 5.18; N, 9.88.

\section{Conclusions}

A novel synthetic modification of the pyrimidine and quinazoline systems involves a selective conjugate addition reaction of various $N$-, $O-, S$-, and $C$-centered nucleophiles across the vinyl function of 2-chloro-4-vinylpyrimidine, 2-chloro-4-(1-phenylvinyl)pyrimidine, and 2-chloro-4-vinylquinazoline followed by nucleophilic displacement of chloride in the resultant intermediate products.

\section{References and Notes}

1. Ishida, A.; Uesugi, T.; Takamuku, S. Photoinduced Alkoxylation of 2-Vinylpyridinium Ion. Bull. Chem. Soc. Jpn. 1993, 66, 1580-1582.

2. Bhanushali, M.J.; Nandurkar, N.S.; Bhor, M.D.; Bhanage, B.M. Cation exchange resin catalyzed hydroamination of vinylpyridines with aliphatic/aromatic amines. Catal. Comm. 2008, 9, 425-430; references cited therein.

3. Schaaf, G.M.; Mukherjee, S.; Waterson, A.G. Conjugate addition of sodium methanesulfinate to vinyl pyridines and diazines for the synthesis of aliphatic sulfones. Tetrahedron Lett. 2009, 50, 1928-1933.

4. Gill, A.L.; Frederickson, M.; Cleasby, A.; Woodhead, S.J.; Carr, M.G.; Woodhead, A.J.; Walker, M.T.; Congreve, M.S.; Devine, L.A.; Tisi, D.; O’Reilly, M.; Seavers, L.C.A.; Davis, D.F. Identification of novel p38alpha MAP kinase inhibitors using fragment-based lead generation. $J$. Med. Chem. 2005, 48, 414-426.

5. Samaritoni, J.G.; Babbitt, G.E. Synthesis of 2-(4-quinazolinyl)ethyl sulfides via addition of thiols to 4-vinylquinazolines. J. Heterocycl. Chem. 1997, 34, 1263-1266.

6. Sączewski, J.; Paluchowska, A.; Klenc, J.; Raux, E.; Barnes, S.; Sullivan, S.; Duszyńska, B.; Bojarski, A.J.; Strekowski, L. Synthesis of 4-substituted 2-(4-methylpiperazino)pyrimidines and quinazoline analogs as serotonin 5- $\mathrm{HT}_{2 \mathrm{~A}}$ receptor ligands. J. Heterocycl. Chem. 2009, 46, 1259-1265.

7. Krištafor, S.; Gazivoda-Kraljević, T.; Makuc, D.; Plavec, J.; Šuman, L.; Kralj, M.; Raić-Malić, S. Synthesis, Structural Studies and Antitumoral Evaluation of C-6 Alkyl and Alkenyl Side Chain Pyrimidine Derivatives S. Molecules 2009, 14, 4866-4879.

8. Mokrosz, M.J.; Strekowski, L.; Kozak, W.X.; Czarny, A.; Duszyńska B.; Bojarski, A.J.; Mokrosz, J.L. Structure-activity relationship studies of CNS agents. Part 25: 2-(N-Methylpiperazino)-4,6di(heteroaryl)pyrimidines as new potent 5- $\mathrm{HT}_{2 \mathrm{~A}}$ receptor ligands: A verification of the topographic model. Arch. Pharm. (Weinheim) 1995, 328, 659-666. 
9. Mokrosz, J.L.; Strekowski, L.; Duszyńska B.; Harden, D.B.; Mokrosz, M.J.; Bojarski, A.J. Structure-activity relationship studies of CNS agents. Part 14. Structural requirements for the 5$\mathrm{HT}_{1 \mathrm{~A}}$ and $5-\mathrm{HT}_{2}$ receptor selectivity of simple 1-(2-pyrimidinyl)piperazine derivatives. Pharmazie 1994, 49, 801-806.

10. Mokrosz, J.L.; Duszyńska, B.; Charakchieva-Minol, S.; Bojarski, A.J.; Mokrosz, M.J.; Wydra, R.L.; Janda, L.; Strekowski, L. Structure-activity relationship studies of CNS agents. Part 29. NMethylpiperazino-substituted derivatives of quinazoline, phthalazine and quinoline as novel $\alpha_{1}, 5$ $\mathrm{HT}_{1 \mathrm{~A}}$ and 5-HT $2 \mathrm{~A}$ receptor ligands. Eur. J. Med. Chem. 1996, 31, 973-980.

11. Lipshutz, B.H.; Sengupta, S. Organocopper reagents: Substitution, conjugate addition, carbo/metallocupration, and other reactions. Org. React. 1992, 41, 146-148.

12. Warwicker, J.; Gane, P.J. Calculation of Cys $30 \Delta \mathrm{pK}_{\mathrm{a}}$ 's and oxidising power for DsbA mutants. FEBS Lett. 1996, 385, 105-108.

13. Bartlett, P.; Meadows, J.; Ottow, E. Enantiodivergent syntheses of (+)- and (-)-nonactic acid and the total synthesis of nonactin. J. Am. Chem. Soc. 1984, 106, 5304-5311.

Sample Availability: Samples of the compounds 5-12, 15-17, and 20-26 are available from the authors.

(C) 2010 by the authors; licensee Molecular Diversity Preservation International, Basel, Switzerland. This article is an open-access article distributed under the terms and conditions of the Creative Commons Attribution license (http://creativecommons.org/licenses/by/3.0/). 